\title{
ONDAS E ISLAS DE CALOR REGISTRADOS EN SANTIAGO DE CHILE EN ENERO DE 2017
}

\author{
Hugo Iván Romero Aravena ${ }^{(a)}$ Dustyn Opazo ${ }^{(b)}$ \\ (a) Departamento de Geografía, Facultad de Arquitectura y Urbanismo de la Universidad de Chile, Santiago de \\ Chile hromer@uchilefau.cl \\ (b)Departamento de Geografía, Facultad de Arquitectura y Urbanismo de la Universidad de Chile, Santiago de \\ dustyn.opazo@gmail.com
}

\section{Eixo: CLIMATOLOGIA EM DIFERENTES NÍVEIS ESCALARES: MUDANÇAS E VARIABILIDADES}

\begin{abstract}
Resumen
En enero de 2017, Chile Central fue afectado por una intensa ola de calor, que causó el peor incendio forestal en sus áreas rurales, elevado disconfort térmico en sus ciudades y una generalizada preocupación respecto a la capacidad institucional del país para enfrentar estos tipos de eventos climáticos extremos y sus adversos impactos ambientales. Las tablas y mapas presentan la distribución espacial y temporal de las temperaturas registradas en las Zonas Climáticas Locales que corresponden a algunos de los barrios de la ciudad de Santiago, permitiendo identificar áreas en que se deben implementar medidas de planificación y diseño urbano que mitiguen los efectos de estos eventos extremos en el futuro.
\end{abstract}

Palabras clave: ondas de calor, islas de calor, climas urbanos, Santiago de Chile

\section{Introducción}

Durante el mes de enero de 2017, diferentes ciudades y regiones del Centro-Sur de Chile fueron afectadas por ondas de calor extremo. El día miércoles 25 de enero de 2017, la estación meteorológica ubicada en el centro de Santiago, registró a las 16,31 horas una temperatura máxima de $37,4^{\circ} \mathrm{C}$, que se constituyó en el valor más elevado observado históricamente en dicho lugar y ocurrió como parte de dos ondas de calor: la primera de cinco días de duración -entre el 17 y 21 de enero-, y la segunda de cuatro días, entre el 24 y 27 de enero. Durante esos días sucesivos, las temperaturas máximas diarias registraron valores superiores a $32,4^{\circ} \mathrm{C}$ (tabla 1 y figura 1 ), que es el umbral establecido por la Dirección Meteorológica de Chile como indicador de la presencia de una onda de calor en la ciudad de Santiago, en la medida que tal temperatura se supere a lo menos durante tres días consecutivos. En este caso, los tres días que separan ambos eventos también registraron temperaturas máximas superiores a los $30^{\circ} \mathrm{C}$, aumentando la percepción de estar en condiciones extraordinariamente cálidas y persistentes, lo cual ha sido inmediatamente atribuido al Cambio Global del Clima, sin que se presenten pruebas al respecto. 
Las ondas de calor pueden ser consideradas eventos extremos excepcionales en Santiago, debido al efecto moderador sobre las condiciones térmica que implica su cercanía a la Cordillera de los Andes y la influencia climática de las frías aguas de la costa Pacífica y de la Corriente de Humboldt, ubicada a tan solo cien km de distancia de la ciudad. Hasta ahora han sido días individuales o pares de días de altas temperaturas los que han predominado en el verano de Santiago, como sucedió el 9 y 10 de febrero de 2012, 28 y 29 de diciembre de 2013, 2 y 3 de febrero de 2013 y 12 y 13 de febrero de 2015. Otros eventos de más de tres días seguidos con temperaturas que superan el umbral de $32,4^{\circ} \mathrm{C}$ se han registrado entre el 3 y 6 de enero de 2012 y 15-17 de enero de 2016, además de los eventos de enero de 2017.

La más reciente y prolongada onda de calor, así como la extensa zona geográfica que ha abarcado, se ha constituido en una sorpresiva perturbación ambiental que ha actuado como causa relevante en la ocurrencia de desastrosos incendios forestales en las áreas rurales y en la destrucción de asentamientos poblados, que junto al disconfort térmico en las ciudades se han traducido en importantes riesgos ambientales. Todo ello generó una dramática sensación de desatención y abandono por parte de la sociedad chilena en general, incluyendo a las autoridades de gobierno que anunciaron estar superadas por los acontecimientos, la población afectada que reclamaba acciones más específicas y actores sociales que cuestionaban la calidad de las instituciones encargadas de proteger los bosques y asentamientos poblados, generando una especial situación de alcances políticos muy semejante a la vivida con motivo del terremoto y tsunami que también afectó al centro de Chile el 27 de febrero de 2010 (Romero at al.,2010,; Romero, 2014). Estas ocasiones que politizan los temas ambientales y manifiestan con claridad los alcances sociales y políticos de los desastres socionaturales, constituyen oportunidades para que la geografía física latinoamericana participe activamente en la introducción de reformas educacionales, cívicas e institucionales que son imprescindibles para que estos eventos no continúen repitiéndose.

Para autores como Popke (2016) Hulme (2015, 2009 y 2007), Tesuro (1938/1985) y Baek (2010), es necesario discutir tanto el concepto de clima en general, de los climas urbanos en particular y de los cambios de clima, principalmente ante las declaraciones retóricas que olvidan que el clima y sus cambios forman parte de la vida cotidiana de la sociedad y que su desempeño depende de los tipos y usos del suelo, topología específica de las ciudades, diseños y formas urbanos, materialidades de las construcciones, y muchas otras decisiones sociales que se ejecutan día a día sin advertir sus consecuencias en los plazos largos y medianos y en las necesarias formas de adaptación de las sociedades a climas y culturas siempre cambiantes. 


\section{OS DESAFIOS DA GEOGRAFIA FÍSICA NA FRONTEIRA DO CONHECIMENTO Instituto de Geociências - Unicamp Campinas - SP \\ 28 de Junho à 02 de Julho de 2017}

\section{Resultados}

La tabla 1 presenta las temperaturas máximas registradas durante los días de la onda de calor en un conjunto de estaciones "urbanas" instaladas con fines de investigación científica en la ciudad de Santiago.

Tabla 1. Diferencias entre las temperaturas máximas diarias registradas entre la Estación Quinta Normal y un conjunto de puestos de registros urbanos entre el 17 y 26 de enero de 2017.

\begin{tabular}{|c|c|c|c|c|c|c|c|c|c|c|c|}
\hline & $\begin{array}{l}\text { Quinta } \\
\text { Norma } \\
\text { I DMC }\end{array}$ & $\begin{array}{c}\text { Estació } \\
\text { n } \\
\text { Quinta } \\
\text { Normal }\end{array}$ & $\begin{array}{c}\text { Estació } \\
\text { n } \\
\text { Pirque }\end{array}$ & $\begin{array}{c}\text { Estació } \\
\text { n } \\
\text { Recolet } \\
\text { a }\end{array}$ & $\begin{array}{c}\text { Estación } \\
\text { San } \\
\text { Bernard } \\
\text { o }\end{array}$ & $\begin{array}{c}\text { Estació } \\
\text { n } \\
\text { Renca }\end{array}$ & $\begin{array}{c}\text { Estació } \\
\mathbf{n} \\
\text { Santiag } \\
0\end{array}$ & $\begin{array}{c}\text { Estació } \\
\text { n La } \\
\text { Florida }\end{array}$ & $\begin{array}{c}\text { Estació } \\
\text { n } \\
\text { Maipú }\end{array}$ & $\begin{array}{c}\text { Estació } \\
\text { n San } \\
\text { Miguel }\end{array}$ & $\begin{array}{c}\text { Estació } \\
\mathbf{n} \\
\tilde{\text { Nuñ̃oa }}\end{array}$ \\
\hline $\begin{array}{c}17 / 01 / 201 \\
7\end{array}$ & 34,8 & 1,01 & $-1,53$ & $-0,28$ & $-0,61$ & 0,26 & $-0,13$ & $-1,03$ & $-0,26$ & $\mathbf{- 0 , 7 7}$ & $-0,28$ \\
\hline $\begin{array}{c}18 / 01 / 201 \\
7\end{array}$ & 36,2 & 0,8 & $-1,68$ & $-0,31$ & $-0,82$ & $\mathbf{0 , 3 3}$ & $\mathbf{0 , 0 8}$ & $-1,14$ & $-0,39$ & $-0,84$ & $-0,47$ \\
\hline $\begin{array}{c}19 / 01 / 201 \\
7\end{array}$ & 35,5 & $\mathbf{0 , 5 4}$ & $-1,42$ & $-0,31$ & $-0,65$ & $\mathbf{0 , 3 1}$ & $-0,07$ & $-0,92$ & $-0,28$ & $-0,72$ & $-0,34$ \\
\hline $\begin{array}{c}20 / 01 / 201 \\
7\end{array}$ & 36,9 & 0,25 & $-1,89$ & 0,15 & $-0,45$ & 0,02 & $-0,1$ & $-1,46$ & $-0,52$ & $-1,23$ & $-\mathbf{0 , 3 8}$ \\
\hline $\begin{array}{c}21 / 01 / 201 \\
7\end{array}$ & 34,2 & 0,67 & $-1,27$ & $-0,23$ & $\mathbf{0 , 5 2}$ & $-0,12$ & 0,19 & $-0,87$ & 0,17 & $-0,81$ & $-0,3$ \\
\hline $\begin{array}{c}24 / 01 / 201 \\
7\end{array}$ & 34,2 & $\mathbf{0 , 5 2}$ & $-1,18$ & $-0,26$ & 0,28 & 0,08 & 0,11 & $-1,11$ & 0,23 & $-0,79$ & $-0,35$ \\
\hline $\begin{array}{c}25 / 01 / 201 \\
7\end{array}$ & 37,4 & 0,12 & $-1,71$ & $-0,02$ & $-0,71$ & $-0,14$ & $-0,38$ & $-1,72$ & $-0,61$ & $-1,45$ & $-0,66$ \\
\hline $\begin{array}{c}26 / 01 / 201 \\
7\end{array}$ & 33,4 & 0,26 & $-0,92$ & $\mathbf{0 , 3 2}$ & $-0,66$ & 0,22 & $-0,02$ & $-0,92$ & $-0,33$ & $-0,81$ & $-0,19$ \\
\hline $\begin{array}{c}27 / 01 / 201 \\
7\end{array}$ & 34,3 & 0,44 & $-1,03$ & $-0,29$ & $-0,8$ & 0,15 & $-0,16$ & $-1,03$ & $-0,41$ & $-0,88$ & $-0,22$ \\
\hline
\end{tabular}

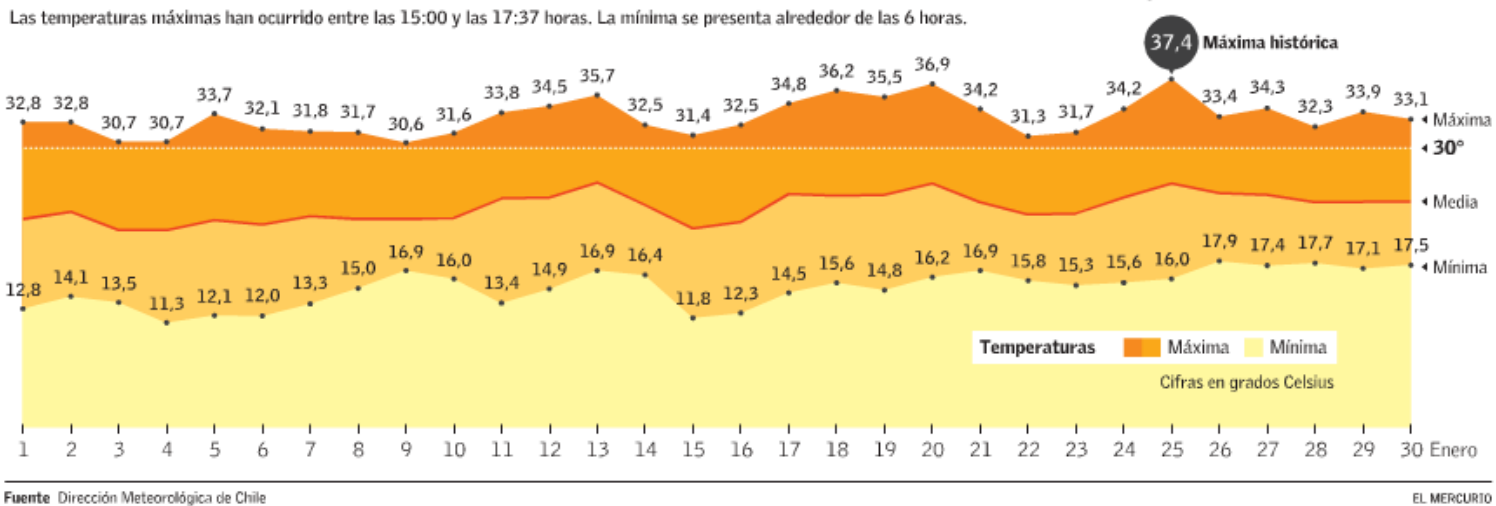

Figura 1: Temperaturas máximas, medias y mínimas registradas en Quinta Normal, Santiago, en enero de 2017. Fuente: Dirección Meteorológica de Chile, publicado por El Mercurio, 31 de enero de 2017, A-12.

El concepto de Zona Climática Local (Stewar and Oke, 2009) (figura 2) corresponde a un área de la ciudad que, en virtud de sus usos del suelo, tipos de construcciones y coberturas vegetales, deberían producir condiciones climáticas distintas a sus zonas vecinas. Tal como se puede apreciar en la figura, existe una gran cantidad de zonas climáticas locales en función del tamaño y complejidad de la ciudad, pero pueden reconocerse algunas áreas principales en virtud de sus rasgos socio-ecológicos predominantes. 




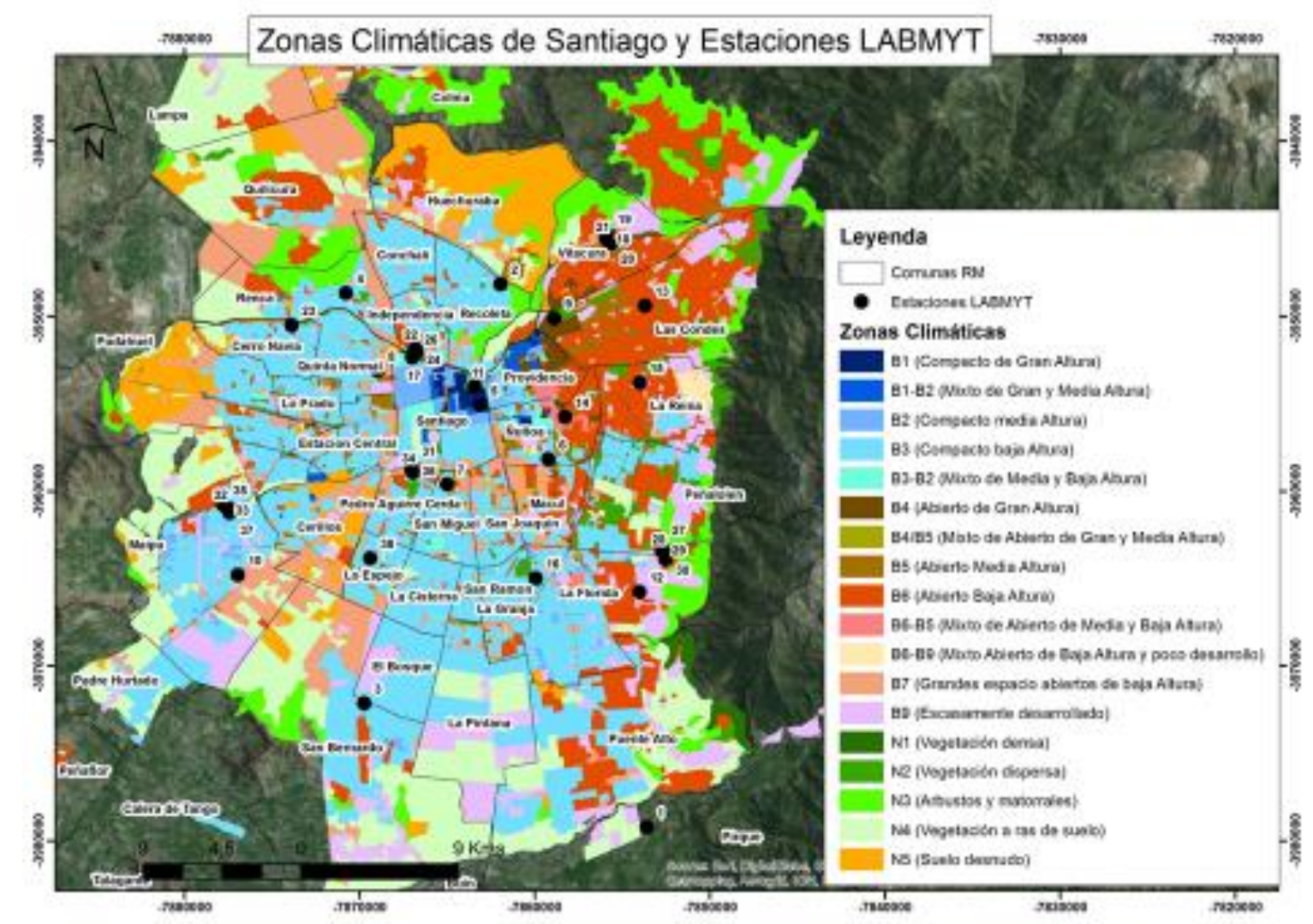

Figura 2. Zonas Climáticas Locales en la ciudad de Santiago

Mapas anteriores elaborados para la estación de verano (Smith y Romero, 2012), han demostrado que las islas de calor urbano son altamente dinámicas, desplazándose diaria y horariamente. Los mapas adjuntos presentan resultados preliminares que contienen datos extremos y promedios para un período de seis años, pudiendo advertirse la presencia de puntos e islas de calor en la ciudad, que deberían ser objeto de tratamientos urbanos específicos tanto para mitigar su existencia, como para evitar su creación, o contrarrestarlas aumentando, complementariamente, las islas frías. 




Figura 3. Temperaturas máximas absolutas registradas en barrios de Santiago 2000-1016

En el caso de las temperaturas máximas y promedio del verano (figura 4), se aprecia una franja de valores altos en el Norte de la ciudad, con centro en la Comuna de Vitacura; una franja menos cálida en el centro, que se extiende por las comunas de Conchalí, Recoleta, Las Condes, Providencia y La Reina; y una franja cálida del centro sur, que incluye las comunas de Maipú, Quinta Normal, Macul y Peñalolén. Esta distribución de las temperaturas más elevadas a través de franjas transversales N-S, complementa la distribución esencialmente oriente-poniente presentada en anteriores trabajos (Smith y Romero, 2012).

La representación espacial presenta diferencias cuando se analizan los datos promedios de la serie (figura 5), destacando en este caso la existencia de un triángulo de temperaturas más elevadas en la zona surponiente de la ciudad, que, al prolongarse hacia el norte, separa dos franjas esencialmente longitudinales $(\mathrm{N}-\mathrm{S})$ que se registran en los sectores oriente y poniente y que están más de acuerdo con anteriores análisis. 
XVII Simpósio Brasileiro de Geografia Fisica Aplicada I Congresso Nacional de Geografia Física

\section{OS DESAFIOS DA GEOGRAFIA FÍSICA NA FRONTEIRA DO CONHECIMENTO}

Instituto de Geociências - Unicamp Campinas - SP

28 de Junho à 02 de Julho de 2017

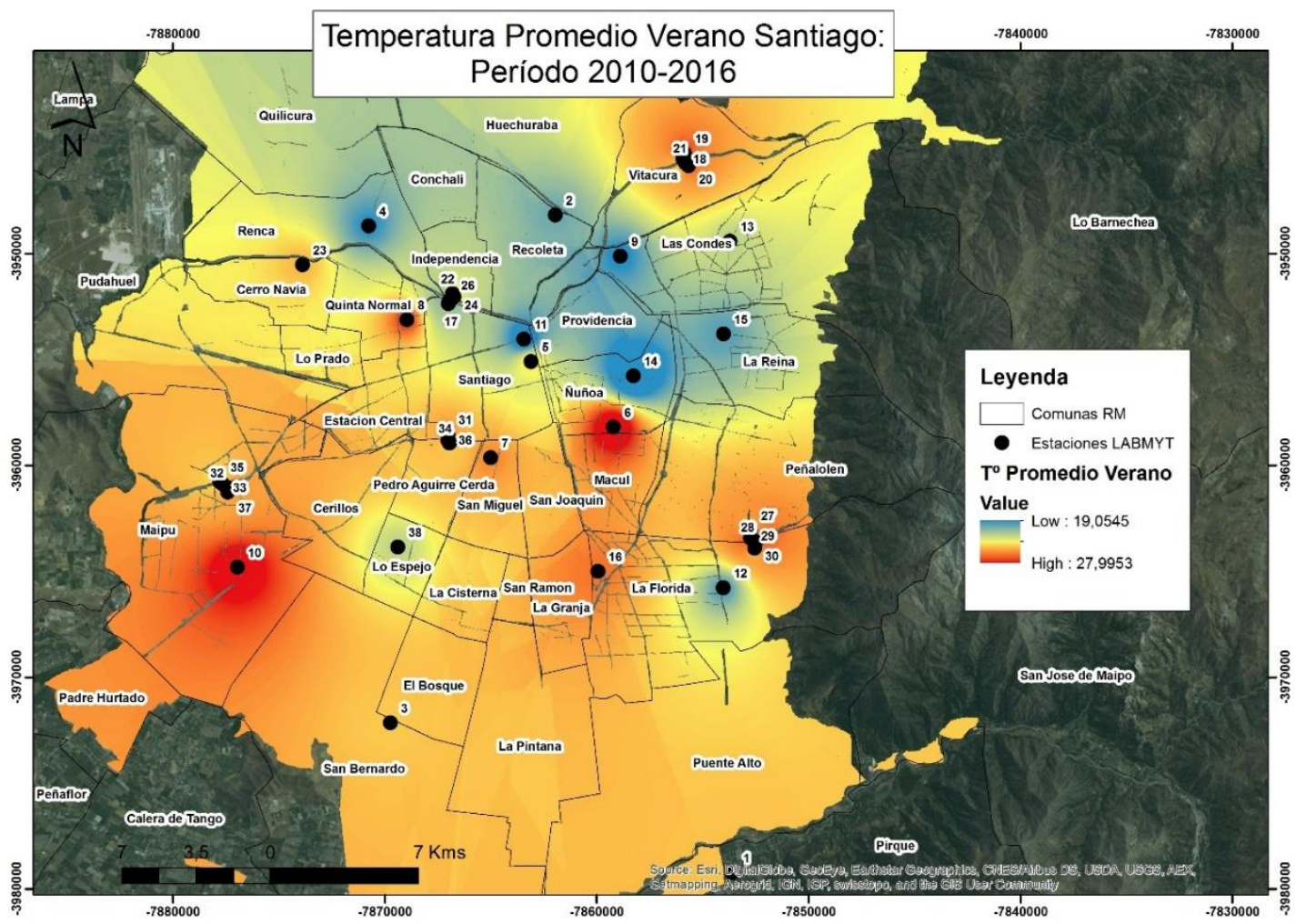

Figura 4. Temperaturas máximas promedio en los barrios de Santiago 2000-2016



Figura 5: Temperaturas promedio de los barrios de Santiago 2000-2016 


\section{OS DESAFIOS DA GEOGRAFIA FÍSICA NA FRONTEIRA DO CONHECIMENTO \\ Instituto de Geociências - Unicamp \\ Campinas - SP \\ 28 de Junho à 02 de Julho de 2017}

Finalmente, la tabla 4 presenta las diferencias entre las temperaturas registradas en puntos urbanos de los barrios de Santiago y en Quinta Normal en las ondas de calor de los meses de enero de 2012, 2014, 2015 y 2017 . El barrio de la comuna de Santiago registró una diferencia positiva de $8,7^{\circ} \mathrm{C}$ y el de San Miguel de $4,0^{\circ} \mathrm{C}$ el 3 de enero de 2012. Este último punto superó a la estación de referencia en $6,3^{\circ} \mathrm{C}$ el 5 de enero de 2012. Este mismo día Maipú superaba en $4,4^{\circ} \mathrm{C}$ a Quinta Normal.

En la onda de calor del 11, 12 y 13 de enero de 2014, los dos primeros días las diferencias fueron más moderadas e incluso negativas, indicando que barrios como Renca y Ñuñoa dichos días registraron temperaturas menores en $3^{\circ} \mathrm{C}$ en comparación a Quinta Normal.

En la onda de calor del 9 al 11 de enero de 2015 se observa un cambio significativo entre el primer y último día. En el primer caso, las temperaturas de los barrios son más frías, pudiendo superar $-5^{\circ} \mathrm{C}$ de diferencia entre Nuñoa y Quinta Normal. Sin embargo, el último día de ese evento cálido, la temperatura de los barrios fue superior en más de $2^{\circ} \mathrm{C}$ en Renca, Santiago y Maipú y alcanzó a $+6,39^{\circ} \mathrm{C}$ en Recoleta.

En las ondas de calor de 2017, se advierte una generalización espacial que abarca casi la totalidad de la ciudad. Sólo Pirque, La Florida y San Miguel registran temperaturas máximas alrededor de un grado menos que Quinta Normal.

Tabla 3. Diferencias de temperaturas registradas entre puntos de barrios de Santiago y Quinta Normal en las ondas de calor de los años 2012, 2014 y 2015

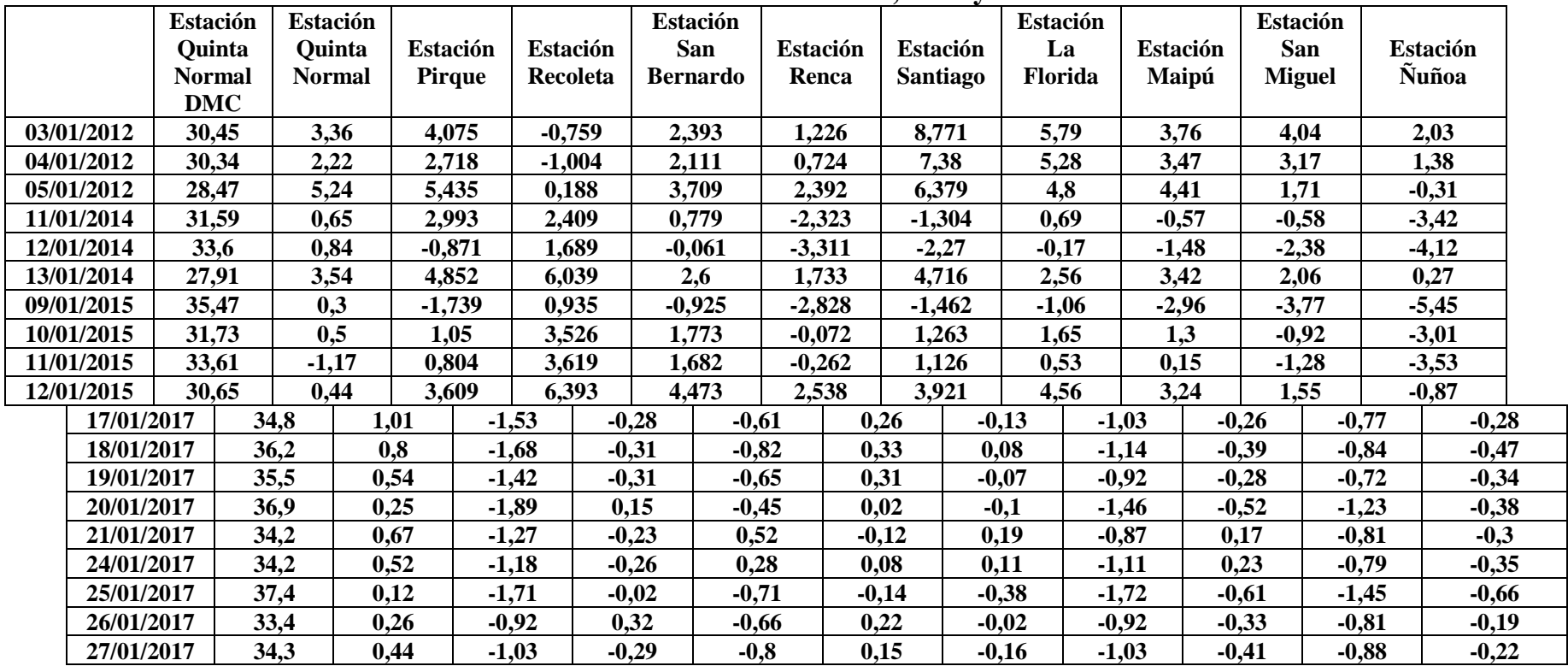

\section{Conclusiones}

Los climas urbanos son un complejo mosaico de Zonas Climáticas Locales e islas de calor que no son necesariamente representados por los datos obtenidos en las estaciones estandarizadas que informan a 
la sociedad sobre eventos extremos, tales como las ondas de calor. Los diferentes barrios y al interior de ellos, las zonas climáticas locales, conforman áreas de diferencias significativas, que deberían ser tomadas en cuenta al proponer medidas que mitiguen los efectos del calor urbano. En la última década, las ondas de calor han aumentado en duración y magnitud, hasta culminar con el episodio de 2017. Es importante proponer medidas de mitigación y adaptación de las ciudades bajo condiciones de extremos térmicos (cálidos o fríos) para evitar la repetición de desastres como los que han afectado a Europa.

Los tiempos y espacios de variaciones son múltiples. Existen diferencias entre los puntos urbanos a todas las escalas temporales y una misma onda de calor puede resultar en días más cálidos o más fríos en algunos de los barrios. La existencia de una red de mediciones es imprescindible en la medida que se reconozca que los climas urbanos son construcciones sociales y no hechos de la naturaleza y que, por lo tanto, corresponde a la sociedad evitar que se transformen en desastres.

\section{Bibliografía}

-BAEK, J. (2010). Climate, Sustainability and The Space Of Ethics, Architectural Theory Review, 15:3, 377395.

-HULME, M. (2007). Geographical work at the boundaries of climate change. Transactions of the Institute of British Geographers 33:5-11.

-HULME, M. (2009). Cosmopolitan Climates: hybridity, foresight and meaning. Theory, Culture and Society on Global Heating: social theory looks at climate change.

-HULME, M. (2015). Climate and its changes: a cultural appraisal. Geo: Geography and Environment, published by John Wilwy and Sons.

-POPKE, J. (2016). Researching the hybrid geographies of climate change:reflections from the field. Area, 2016, 48, 1: 2-6.

-ROMERO, H. -"Vulnerabilidad, resiliencia y ordenamiento territorial de los desastres socionaturales en Chile". Revista Polígonos, España, 26 (2014): 87-110, Publicación de las universidades de Valladolid, Salamanca y Castilla La Mancha.

-ROMERO, H., FUENTES, C. y SMITH, P. "Dimensiones geográficas territoriales, institucionales y sociales del terremoto de Chile de febrero 2010". Cuadernos de Geografía, Revista Colombiana de Geografía. Bogotá: Universidad Nacional de Colombia, $\mathrm{n}^{\circ}$ 19, 2010, p. 135-150.

-SMITH, P. y ROMERO, H. (2016). Construcción de un modelo para estudiar la distribución termal urbana en Santiago de Chile. Revista de Geografía Norte Grande 63:45-62

-STEWART, I.D. \& OKE, T. (2009), Classifying urban climate field sites by local climate zones: The case of Nagano, Japan. In: Preprint, Seventh International Conference on Urban Climate, 29 june -3 july 2009, Yokohama.

-TESURO, W. (1988/1935). Climate and Culture: A philosophical study (Trans Bownas, G.) Green Wood Press, New York. 